\title{
A literacia da informação no combate às fake news: desafios e estratégias formativas no ensino superior
}

\author{
Maria da Luz Antunes ${ }^{1,2}$, Carlos Lopes ${ }^{2,3}$, Tatiana Sanches ${ }^{2,4,5}$ \\ ${ }^{1}$ ORCID 0000-0003-0942-7601 + Escola Superior de Tecnologia da Saúde de Lisboa (Instituto \\ Politécnico de Lisboa), Lisboa, Portugal. mluz.antunes@estesl.ipl.pt \\ 2 APPsyCI - Applied Psychology Research Center Capabilities \& Inclusion, ISPA - Instituto \\ Universitário, Lisboa, Portugal. \\ ${ }^{3}$ ORCID 0000-0002-6440-4739 + ISPA - Instituto Universitário, Lisboa, Portugal. clopes@ ispa.pt \\ ${ }^{4}$ ORCID 0000-0002-4902-2628 + Instituto de Educação (Universidade de Lisboa), Lisboa, \\ Portugal.tsanches@fpie.ulisboa.pt \\ ${ }^{5}$ UIDEF, Instituto de Educação (Universidade de Lisboa), Lisboa, Portugal.
}

\section{Resumo}

Introdução: Os problemas com a verdade e a falácia na Internet têm vindo a evidenciar-se, tornando-se num mar de navegação perigosa devido à quantidade de falsas verdades, desinformação, informação enganosa ou errónea - um conjunto de conceitos agregados no que é mais conhecido por fake news. O presente estudo argumenta que é necessário recentrar as preocupações com a informação no elemento humano, desenvolvendo intervenções educativas para alterar esta realidade, consciencializando e formando os utilizadores da informação. Especificamente para o combate às fake news, as instituições de ensino superior devem capacitar a sua comunidade para uma navegação segura na Internet, ensinando-a a filtrar os conteúdos que irão utilizar, colocar e disponibilizar para outrem. Estudantes, professores e investigadores devem saber qual a informação de que necessitam, conseguir identificar o que procuram, reconhecer as condições sob as quais a informação pode ser reutilizada de forma ética, assim como o destino que terá, e distinguir entre conhecimento, opinião e comentário. O objetivo do presente estudo é o de conhecer a atuação dos profissionais da informação no combate às fake news, através da análise das estratégias em competências de literacia da informação em contexto académico. Método: Realizouse uma revisão da literatura indexada na Scopus e na Web of Science, associando fake news e a literacia da informação no contexto do ensino superior. Resultados: A Web of Science apresenta um total de 41 resultados e a Scopus de 22 resultados. A análise aponta para a descrição de iniciativas e projetos oriundos quer de bibliotecas do ensino superior, quer de profissionais da informação do mesmo setor, comprometidos com a causa do combate às fake news. Discussão: A literatura refere que o fator educacional deve necessariamente ser ponderado: a formação de utilizadores motivados para o conhecimento é que lhes permite distinguir a veracidade do que se afirma e identificar qual o cenário para a produção de mais conhecimento. As pessoas melhor preparadas correspondem a indivíduos que assumem como insuficiente a informação disponibilizada pela Internet. As bibliotecas do ensino superior assumem-se como importantes núcleos neste processo: porque se formam e porque formam, mantendo-se atuais e confiáveis. Sugere-se o desenvolvimento de medidas a implementar pelas instituições de ensino superior e pelos profissionais da informação para um eficaz combate às fake news, em especial no contexto académico. Concluiu-se que o conhecimento pode resultar em informação, mas a informação não resulta necessariamente em conhecimento - e a informação pode não ultrapassar o patamar da opinião, pelo que importa reforçar estratégias formativas. 
Palavras-chave: Literacia da informação; Fake news; Ensino superior; Estratégias formativas; Aprendizagem.

Las noticias falsas no se combaten con la censura sino precisamente con más noticias y más formación en fuentes de información. (López-Borrull, Vives-Gràcia, \& Badell, 2018, p. 1354).

\section{o Contexto de Partida}

Em agosto de 2018, a International Federation of Libraries Associations (IFLA, 2018) elaborou uma Declaração sobre as fake news, onde manifestou a sua profunda preocupação pelo impacto público desproporcionado que o fenómeno das fake news pudesse assumir face à liberdade de expressão e ao acesso à informação. Nesta Declaração é reforçado o compromisso, desde sempre assumido pela IFLA, sobre a liberdade de acesso à informação e à liberdade de expressão. Deixa claro que qualquer restrição a esta liberdade, mediante a censura ou o bloqueio de acesso aos recursos digitais e à Internet, deve ser limitada, o que inclui respeitar o Princípio de Manila sobre a Responsabilidade dos Intermediários (https://www.manilaprinciples.org/pt-br).

A IFLA desafia os governos a investir em programas de literacia da informação e literacia mediática, a todos os níveis e para pessoas de todas as idades, que respondam às necessidades atuais, em especial através das bibliotecas e das escolas, como parte de projetos mais amplos para lograr atingir os objetivos em educação da Agenda 2030 da ONU.

Com base neste contexto procura-se, através de uma revisão da literatura, dar uma resposta sobre o papel das bibliotecas de ensino superior e dos seus profissionais no combate às fake news através da literacia da informação, de modo a ajudar a comunidade académica a avaliar de forma crítica a credibilidade e a pertinência das fontes de informação.

\section{Introdução}

Vive-se na atualidade numa era em que qualquer internauta é também um produtor de conteúdos e um difusor de informação. Mas também se vive numa era de pós-verdade, caracterizada por factos que são paulatinamente substituídos por crenças e a natureza da verdade modificada por

emoções. É, então, necessário recentrar as preocupações com a informação no elemento humano. A autonomia e liberdade digitais ao nível individual trouxeram consigo mais responsabilidade cívica e social, tornando prementes as questões éticas e legais associadas à pesquisa, utilização e disponibilização da informação (López-Borrull, Vives-Gràcia, \& Badell, 2018).

Desde o ano 2000 que os problemas com a verdade e a falácia na Internet se tornaram um tema de debate, sendo que a preocupação aumentou nos anos mais recentes. Devido à incúria, inocência ou falta de preparação dos internautas, dados imprecisos podem desencadear perigos diversos, afetando a sua saúde, privacidade, os seus investimentos, decisões de negócios, compras online e mesmo matérias legais. A Internet tornou-se um mar de navegação perigosa devido à quantidade de falsas verdades, desinformação, informação enganosa ou errónea, sob uma infinidade de formas e disfarces (Forbes \& Mintz, 2002). Todos os dias surgem novas mentiras e camuflagens que são usadas para enganar os mais incautos, designadamente no que se refere ao roubo de identidade digital, apelo ao ódio, intolerância religiosa e racial, fraude nas transações de $e$-commerce, questões 
de cibersegurança, fraude política, de caridade, entre outras (Mintz, 2012). Assim, é cada vez mais importante e urgente um comportamento preventivo face a estes riscos.

As fake news não são um fenómeno novo: Pulitzer usava manchetes sensacionalistas e George Orwell, em 1984, relatava a destruição de documentos pelos líderes partidários para eliminar as provas da mentira. Mas o método atual parece ser mais eficiente: ao invés de destruir os factos, fabrica-se uma história, a qual é continuamente repetida até que seja interpretada como real e apresentada como um produto das tecnologias da informação. Constata-se, desde 2016, que nas redes sociais as notícias são partilhadas como um marcador de identidade; partilham-se notícias não só para informar como para persuadir outrem; partilham-se notícias para evidenciar dedicação a uma comunidade, a um sentimento ou a uma ideologia. $\mathrm{O}$ intuito desta partilha é o fortalecimento de laços comunitários.

Como intervir para alterar o panorama? É importante saber que a disseminação de notícias falsas ou verdadeiras tem a mesma rapidez ou velocidade quando são analisadas as redes sociais e o seu impacto na difusão de informação. Porém, o elemento humano altera esta equação: "false news spreads farther, faster, deeper, and more broadly than the truth, because humans, not robots, are more likely to spread it" (Vosoughi, Roy, \& Aral, 2018, p. 1148). Esta constatação revela uma importante pista. É que podem ser levadas a cabo intervenções educativas para alterar esta realidade, consciencializando e formando utilizadores de informação no seu bom uso.

A atenção dada a esta problemática ilustra também um fluxo natural entre os temas da sociedade civil e a academia. No meio científico, a procura de informação é uma necessidade incontornável. E a Internet não é suficiente quando se investiga, porque a investigação assenta em informação e em dados fidedignos, completos e credíveis. A Ciência Aberta tem proporcionado uma expansão dos territórios de investigação, dando uma resposta mais adequada a estas necessidades. Ao libertar dados e informação de uma esfera restrita para a esfera pública e fazendo circular maior quantidade de conhecimento, potencia a utilização da ciência por gamas mais alargadas de público. Ao mesmo tempo, se a procura de informação não tem as suas fronteiras tão bem definidas, a navegação pode ocorrer em contextos digitais arriscados, revelando-se uma questão particularmente sensível (Jasanoff, 2016). As redes sociais são mais propícias a essas ocorrências e têm sido, aliás, fonte e objeto de investigação.

As questões que se apresentam às instituições de ensino, em especial as do ensino superior, são, deste modo, muito abrangentes: é necessário capacitar as pessoas para navegarem de forma segura na Internet, mas é também essencial ensiná-las a filtrar os conteúdos que irão utilizar ou colocar e disponibilizar para outrem, porque a Internet se tornou o recurso privilegiado para a pesquisa de informação em ambiente académico e porque os estudantes preferem a informação alojada em recursos fáceis de localizar e de aceder, ao invés de privilegiar os critérios de qualidade da informação (Cid-Leal \& Perpinyà-Morera, 2015). Nesta linha foram publicados muito recentemente diversos estudos que reforçam o papel dos profissionais da informação e das bibliotecas (Fontanin, 2018; Gilchrist, 2018; Rose-Wiles, 2018).

Diversos organismos internacionais elencaram um conjunto de tendências para as bibliotecas académicas, enquanto modelos observáveis dos contextos de atuação e da forma como aquelas se devem preparar para o futuro próximo. Estes organismos, que procuram pensar sobre si próprios, analisar o contexto e as comunidades que representam e apontar caminhos para as bibliotecas que inspiram (num progressivo contributo para o prosseguir das missões de ensino e investigação nas instituições de que fazem parte), assumem também que, na sua origem, as bibliotecas foram 
estabelecidas sob a égide de que a informação é um bem social. Durante a $2^{\mathrm{a}}$ Guerra Mundial, face ao futuro da democracia, Roosevelt descreveu as bibliotecas como sendo os grandes símbolos da liberdade, considerando-as essenciais para o funcionamento de uma sociedade democrática (Johnson, 2017).

Em 2018, a UNESCO manifestou a sua preocupação sobre este fenómeno viral das fake news ao publicar o manual Journalism 'fake news' and disinformation. Escrito por especialistas na luta contra a desinformação, o manual explora a natureza do próprio jornalismo com módulos sobre porque é importante a confiança; reflete criticamente sobre a tecnologia digital e as plataformas das redes sociais como vetores de comportamentos de desordem da informação; aborda a luta contra a desinformação através da literacia mediática e da informação; e incide na verificação dos factos, na verificação das redes sociais no combate contra o abuso online. Conclui com a necessidade de sinergias entre os profissionais da comunicação e da informação no combate à manipulação manifesta da opinião pública nas plataformas das redes sociais surgida como uma ameaça crítica para a vida pública e democrática (UNESCO, 2018).

O mais recente cenário apontado pela International Federation of Libraries Associations (IFLA, 2018) serve de exemplo para o combate das bibliotecas às fake news, usando a metáfora dos ataques e pilhagens da Idade Média às bibliotecas e referindo que hoje, ao contrário da defesa e guarda, interessa usar a arma da difusão e transmissão do conhecimento. É também reforçada a ideia de que as bibliotecas se vêm coartadas e ameaçadas em diversas funções, devendo, assim, apostar mais do que nunca em franquear acesso à informação, apoiar o acesso aberto e apostar na sua expertise para transmitir confiança aos utilizadores.

A instituição europeia Ligue des Bibliothèques Européennes de Recherche (LIBER Europe, 2017), por seu turno, identifica direções estratégicas para as bibliotecas do ensino superior no período 2018-2022 ao sublinhar a importância das bibliotecas serem consideradas como plataformas de comunicação académica inovadora, como centros de habilidades e serviços digitais em ambientes de investigação física e virtual, reforçando ainda o posicionamento das bibliotecas como espaços de preservação do património cultural digital e humanidades digitais. A LIBER Europe prevê, por conseguinte, o desenvolvimento de ações de formação nas áreas do copyright, das questões legais, do acesso aberto, das métricas inovadoras, mas também na formação dos profissionais. Face às questões que envolvem as fake news declara que "in the digital environment, the norms and standards regarding ethical behaviour are changing and adapting to technological possibilities. Libraries need to engage in and advocate for developing information ethics in the interests of the research community" (LIBER Europe, 2017, p. 12).

Já a Association of College and Research Libraries (ACRL Research Planning and Review Committee, 2018), no seu documento prospetivo sobre as principais tendências para as bibliotecas académicas, considera relevantes diversos tópicos, sendo as competências de literacia da informação uma das áreas a repensar. Este aspeto destaca a importância da literacia da informação na realidade das fake news, referindo que devem ser realizadas ações de formação para o desenvolvimento de competências de avaliação das fontes de informação e para o desenvolvimento do pensamento crítico (Connaway, Julien, Seadle, \& Kasprak, 2017). São também englobadas formas inovadoras de educação, em que se propõe o desenvolvimento de novas abordagens para ações de formação através de tutoriais com um design mais apelativo, bem como outras formas de literacia como a literacia digital. Outro aspeto a considerar prende-se com as práticas de preservação digital. Será necessário continuar os esforços para a construção de infraestruturas capazes de salvaguardarem a diversidade de recursos digitais e a crescente abertura e natureza 
colaborativa do processo de investigação. Para tornar efetiva a curadoria de dados de investigação, as bibliotecas são instadas a desenvolver infraestruturas que permitam a incorporação e preservação deste tipo de recursos.

A American Librarian Association, por outro lado, num documento marcadamente político, reafirma a importância de combater todas as formas de distorção da verdade informativa, sublinhando, entre outras estratégias de combate, o "critical role of librarians and library workers in all types of libraries in teaching information literacy skills that enable users to locate information and evaluate its accuracy" (ALA, 2017, para. 24; Cooke, 2018).

Face às várias linhas de atuação e de tendência explanadas pelas várias associações internacionais, as bibliotecas têm desenvolvido, implementado e atualizado os conceitos da literacia da informação ao longo dos anos (Finley, McGowan, \& Kluever, 2017). O seu grande público-alvo são os estudantes. De um modo geral, os estudantes são competentes comunicadores das redes sociais, mas desconhecem o potencial de manipulação destas. Estas preocupações não são exclusivas de pais, educadores, professores ou da sociedade em geral. Em Itália, o Ministério da Educação desenvolveu um projeto para as crianças, habilitando-as a tornarem-se «caçadoras de fake news» (Horowitz, 2017). No Reino Unido, o Parliamentary Group on Literacy e o National Literacy Trust organizaram uma comissão de fake news e de ensino de competências de literacia (National Literacy Trust, 2018). Também a Austrália, a Suécia e os países Bálticos têm desenvolvido esforços meritórios no combate à desinformação (Comber \& Grant, 2018). Por seu turno, a Comissão Europeia delineou um conjunto orientador de princípios e objetivos para o combate às fake news $\mathrm{e}$ que abrangem a transparência da origem da informação, a diversidade e a credibilidade da informação, bem como a oferta de soluções inclusivas que passam pela capacitação em competências de literacia (Comissão Europeia, 2018).

Ao nível do ensino superior, e de forma informal, as bibliotecas têm desenvolvido diversos projetos e iniciativas de modo a assegurar a capacitação de estudantes e investigadores e de modo a despertar os cuidados necessários na pesquisa e análise de informação. Exemplos disso são o LibGuides, como $\mathrm{o}$ da Indiana University East' Fake news LibGuide (https://iue.libguides.com/Fakenews) e o da University of Washington Libraries' Evaluating Information: fake news LibGuide (https://guides.lib.uw.edu/research/evaluate/fakenews), fornecendo aos estudantes os recursos necessários e um plano de verificação das fontes antes de as usar, mas também o infográfico da IFLA (How to spot fake news) que, visual e graficamente, apresenta uma estratégia de análise das fontes de informação.

Neste âmbito, importa encarar estes desafios e desenvolver estratégias em literacia da informação para combater as fake news. Estudantes, professores, investigadores, bem como o cidadão comum, devem saber qual a informação de que necessitam, conseguir identificar o que procuram, reconhecer as condições sob as quais a informação pode ser reutilizada de forma ética, assim como o destino que terá, e distinguir entre conhecimento, opinião e comentário. E estas questões podem ser resolvidas através da capacitação destes utilizadores da informação, privilegiando a intervenção no ensino superior.

\subsection{Objetivo do estudo}

O objetivo do presente estudo é o de promover a atuação dos profissionais da informação no combate às fake news, através do conhecimento de estratégias em competências de literacia da informação em contexto académico. 


\section{Método}

Realizou-se uma revisão da literatura associando o impacto das fake news e a dinâmica da literacia da informação no contexto do ensino superior.

A Scopus e a Web of Science foram as bases de dados pesquisadas. Os termos de pesquisa selecionados foram 'fake news' e 'information literacy', usando o filtro keywords. A recolha de dados foi efetuada no mês de maio de 2019, os quais foram transformados em ficheiros (extensão ris) e exportados para o gestor bibliográfico Mendeley, enquanto ferramenta útil para o cruzamento de dados e para a criação de uma lista definitiva de resultados.

\section{Resultados}

A Web of Science apresenta um total de 41 resultados e a Scopus de 22 resultados. Da fusão dos dados resultou um total de 57 artigos (seis artigos estavam indexados nas duas bases de dados em estudo), de que se excluíram dois artigos por não terem abstract incluído e três artigos por terem sido publicados em idiomas desconhecidos para os autores (turco e russo).

A Tabela 1 contempla a identificação dos artigos que cumpriram os critérios de elegibilidade do presente estudo. Os artigos são identificados pelo título, não pelo DOI (digital object identifier), porque nem todos o possuíam. É identificada a base (ou as bases) de dados onde o artigo se encontra indexado. As colunas ' $\mathrm{S}$ ' e ' $\mathrm{N}$ ' referem-se à classificação dos artigos, isto é, se o âmbito do estudo se enquadra, ou não, no ensino superior. Os títulos dos artigos destacados a negrito correspondem aos estudos cuja análise é descrita no presente trabalho.

Tabela 1. Artigos recuperados na revisão da literatura (Scopus + Web of Science)

\begin{tabular}{|c|c|c|c|c|}
\hline TíTULO DO ARTIGO & Scopus & $\begin{array}{l}\text { WEB OF } \\
\text { SCIENCE }\end{array}$ & $\mathbf{S}$ & $\mathbf{N}$ \\
\hline $\begin{array}{l}\text { Lifting the curtain: examining the importance of the shared Foundation IV: curate in the National School Library standards for } \\
\text { learners, school librarians, and school libraries }\end{array}$ & $\mathrm{X}$ & & & $\mathrm{X}$ \\
\hline A corpus of debunked and verified user-generated videos & $\mathrm{X}$ & & & $\mathrm{X}$ \\
\hline An invitation to globalize the information literacy agenda: expanding discourse & $\mathrm{X}$ & & & $\mathrm{X}$ \\
\hline Science audiences, misinformation, and fake news & & $\mathrm{X}$ & & $\mathrm{X}$ \\
\hline Fake news and alternative facts: information literacy in a post-truth era & & $\mathrm{X}$ & & $\mathrm{X}$ \\
\hline A corpus of debunked and verified user-generated videos & & $\mathrm{X}$ & & $\mathrm{X}$ \\
\hline Swedish teenagers' difficulties and abilities to determine digital news credibility & & $\mathrm{X}$ & & $\mathrm{X}$ \\
\hline $\begin{array}{l}\text { Fake images: the effects of source, intermediary, and digital media literacy on contextual assessment of image credibility } \\
\text { online }\end{array}$ & & $\mathrm{X}$ & & $\mathrm{X}$ \\
\hline Good news, bad news, and fake news: going beyond political literacy to democracy and libraries & & $\mathrm{X}$ & $\mathrm{X}$ & \\
\hline Information literacy and libraries in the age of fake news & & $\mathrm{X}$ & & $\mathrm{X}$ \\
\hline Digital media literacy campaign in identifying news & $\mathrm{X}$ & & & $\mathrm{X}$ \\
\hline Fake news: amenaza u oportunidad para los profesionales de la información y la documentación? & $\mathrm{X}$ & $\mathrm{X}$ & $\mathrm{X}$ & \\
\hline The "Collage Effect" - Against filter bubbles: interdisciplinary approaches to combating the pitfalls of information technology & & $\mathrm{X}$ & & $\mathrm{X}$ \\
\hline Post-truth: an outline review of the issues and what is being done to combat it & & $\mathrm{X}$ & & $\mathrm{X}$ \\
\hline Infomediation and post-truth: the role of libraries & & $\mathrm{X}$ & $\mathrm{X}$ & \\
\hline "Save the Pacific Northwest tree octopus": a hoax revisited. Or: how vulnerable are school children to fake news? & $\mathrm{X}$ & $\mathrm{X}$ & & $\mathrm{X}$ \\
\hline Con il pretesto delle false notizie: insegnare il pensiero critico nella scuola italiana a partire da Carol C. Kuhlthau & & $\mathrm{X}$ & & $\mathrm{X}$ \\
\hline Learning to talk back to texts & & $\mathrm{X}$ & & $\mathrm{X}$ \\
\hline The phenomenon of fake in the context of communication practices & & $\mathrm{X}$ & & $\mathrm{X}$ \\
\hline Post-facts: information literacy and authority after the 2016 election & & $\mathrm{X}$ & & $\mathrm{X}$ \\
\hline Reflections on fake news, librarians, and undergraduate research & & $\mathrm{X}$ & $\mathrm{X}$ & \\
\hline Focus on the facts: a news and information literacy instructional program & $\mathrm{X}$ & & $\mathrm{X}$ & \\
\hline Walking the talk: using digital media to develop distance learners' digital citizenship at the Open University (UK) & $\mathrm{X}$ & & $\mathrm{X}$ & \\
\hline Real or fake? Resources for teaching college students how to identify fake news & $\mathrm{X}$ & & $\mathrm{X}$ & \\
\hline Fake news, special libraries and what it means to be American & $\mathrm{X}$ & & & $\mathrm{X}$ \\
\hline Why librarians can't fight fake news & $\mathrm{X}$ & & & $\mathrm{X}$ \\
\hline News, fake news, and critical authority & $\mathrm{X}$ & & & $\mathrm{X}$ \\
\hline $\begin{array}{l}\text { Examining student perceptions of their knowledge, roles, and power in the information cycle: findings from a 'fake news' } \\
\text { event }\end{array}$ & $\mathrm{X}$ & & & $\mathrm{X}$ \\
\hline The partisan brain: an identity-based model of political belief & & $\mathrm{X}$ & & $\mathrm{X}$ \\
\hline The spread of disinformation: why do people believe them and how to combat them. The importance of media literacy & & $\mathrm{X}$ & & $\mathrm{X}$ \\
\hline
\end{tabular}




\begin{tabular}{|c|c|c|c|c|}
\hline The importance of media education in combating fake news & & $\mathrm{X}$ & & $\mathrm{X}$ \\
\hline Cybersecurity for civil society & & $\mathrm{X}$ & & $\mathrm{X}$ \\
\hline Citizen reporters as a hoax prevention & & $\mathrm{X}$ & & $\mathrm{X}$ \\
\hline Educators' perceptions of information literacy and skills required to spot 'fake news' & $\mathrm{X}$ & & & $\mathrm{X}$ \\
\hline Comparing features of fabricated and legitimate political news in digital environments (2016-2017) & $\mathrm{X}$ & & & $\mathrm{X}$ \\
\hline The report of $8^{\text {th }}$ International Symposium 2018 on Information Management in a Changing World & & $\mathrm{X}$ & & $\mathrm{X}$ \\
\hline Fake news judgement: the case of undergraduate students at Notre Dame University-Louaize, Lebanon & $\mathrm{X}$ & $\mathrm{X}$ & & $\mathrm{X}$ \\
\hline Democracy, information, and libraries in a time of post-truth discourse & $\mathrm{X}$ & $\mathrm{X}$ & $\mathrm{X}$ & \\
\hline Young people and their practices of access and consumption of news in social media & & $\mathrm{X}$ & & $\mathrm{X}$ \\
\hline $\begin{array}{l}\text { What do we do about "fake news" and other forms of false information: the roles of the organization of false information, } \\
\text { professional ethics and information literacy }\end{array}$ & $\mathrm{X}$ & & & $\mathrm{X}$ \\
\hline A new research agenda: instructional practices of activists mobilizing for science & & $\mathrm{X}$ & & $\mathrm{X}$ \\
\hline (Re)conceptualizing digital literacies before and a er the election of Trump & & $\mathrm{X}$ & & $\mathrm{X}$ \\
\hline Fact-checking vs. Fake news: confirmation journalism as a tool of media literacy against misinformation & & $\mathrm{X}$ & & $\mathrm{X}$ \\
\hline Calling out fake news on social media: a comparison of literature in librarianship and journalism & $\mathrm{X}$ & & & $\mathrm{X}$ \\
\hline Digital literacy in the era of fake news: key roles for information professionals & $\mathrm{X}$ & & $\mathrm{X}$ & \\
\hline Young people's conceptions of political information: insights into information experiences and implications for intervention & $\mathrm{X}$ & $\mathrm{X}$ & & $\mathrm{X}$ \\
\hline Getting out the truth: the role of libraries in the fight against fake news & $\mathrm{X}$ & $\mathrm{X}$ & $\mathrm{X}$ & \\
\hline Hacking the research library: wikipedia, Trump, and information literacy in the Escape Room at Fresno State & & $\mathrm{X}$ & $\mathrm{X}$ & \\
\hline The speed reading is in disrepute: advantages of slow reading for the information equilibrium & & $\mathrm{X}$ & & $\mathrm{X}$ \\
\hline Posttruth, truthiness, and alternative facts: information behavior and critical information consumption for a new age & & $\mathrm{X}$ & $\mathrm{X}$ & \\
\hline Spreadable spectacle in digital culture: civic expression, fake news, and the role of media literacies in "post-fact" society & & $\mathrm{X}$ & & $\mathrm{X}$ \\
\hline Interactive storytelling to teach news literacy to children & & $\mathrm{X}$ & & $\mathrm{X}$ \\
\hline Social networks and media literacy: how to use safe & & $\mathrm{X}$ & & $\mathrm{X}$ \\
\hline Fake news: belief in post-truth & & $\mathrm{X}$ & & $\mathrm{X}$ \\
\hline Fake news, information literacy, and scholarly communication in social work & & $\mathrm{X}$ & & $\mathrm{X}$ \\
\hline How to spot fake news? & & $\mathrm{X}$ & & $\mathrm{X}$ \\
\hline Bakhtin, Colbert, and the center of discourse: is there no "truthiness" in humor? & & $\mathrm{X}$ & & $\mathrm{X}$ \\
\hline
\end{tabular}

Nota: Assinalados a negrito os artigos analisados no presente estudo.

A análise aponta para a descrição de iniciativas e projetos oriundos quer de bibliotecas do ensino superior, quer de profissionais da informação do mesmo setor, comprometidos com a causa do combate às fake news.

\subsection{Principais tendências nas estratégias de combate às fake news}

O estudo de López-Borrull, Vives-Gràcia e Badell (2018) apresenta numa revisão da literatura uma descrição de iniciativas e projetos, tanto de bibliotecas como de outros setores da educação e da comunicação; demonstra a importância de dispor de tutoriais de formação online, evidenciando as fontes fiáveis de informação face às fake news. É sugerida a divulgação de serviços de verificação de dados e a criação de formas de colaboração com os cidadãos, bem como a organização de workshops para a deteção de fake news. Reforçando o papel educacional das bibliotecas do ensino superior, os autores apelam ao fortalecimento das coleções, especialmente digitais. Na medida em que a maioria das fake news são divulgadas através de recursos de livre acesso, creem que se as bibliotecas incrementarem as suas coleções com publicações subscritas, a probabilidade de divulgação de fake news será incomparavelmente menor - posição também defendida por Lor (2018). Mas porque um projeto desta natureza envolve recursos financeiros difíceis de assegurar e de manter ao longo do tempo, para a definição de políticas de aquisição, López-Borrull, Vives-Gràcia e Badell (2018) sugerem a criação de projetos em comum com outros profissionais, reforçando o conhecimento dos códigos éticos profissionais.

Bibliotecários e outros profissionais da informação têm desempenhado um importante papel no combate às fake news, criando ferramentas para apoio aos estudantes do ensino superior e promovendo o desenvolvimento de competências de literacia da informação e do pensamento crítico. O estudo de Neely-Sardon e Tignor (2018) descreve a experiência dos bibliotecários do Indian River State College (IRSC). Criaram um programa abrangente de literacia da informação, o qual disponibilizam aos estudantes através de cursos de capacitação e a que se atribui um crédito. $\mathrm{O}$ programa de formação em literacia da informação que a biblioteca já disponibilizava focava-se bastante na avaliação das fontes de informação. Face à problemática das fake news, essa 
componente foi ampliada. O programa atual consiste em lições, tarefas, kits de aprendizagem e outros recursos. Os bibliotecários desenvolvem esta atividade presencialmente e em ambiente online; são também convidados para ensinar em sala de aula. Os conteúdos da Framework for Information Literacy for Higher Education (ACRL, 2016) foram revistos e optou-se por continuar a investir na frame da Autoridade, identificada como sendo a mais crítica no atual quadro social e educacional.

No IRSC são os bibliotecários que se encarregam dos cursos de literacia da informação, que são opcionais. As sessões costumam contar com 50 a 100 estudantes. A maioria destas sessões é informal e depende da boa vontade institucional e dos convites de professores. Também a maioria do corpo docente assumiu desconhecer a associação entre literacia da informação e fake news e que os profissionais da informação podiam adaptar os conteúdos da literacia da informação para um eficaz combate à problemática. Para corrigir este equívoco, na Primavera de 2017, os bibliotecários promoveram uma campanha para capacitação do corpo docente sobre as opções da literacia da informação, a sua conexão com as fake news e as ferramentas educacionais para a correta identificação de fontes de informação fidedignas. Demonstrou-se, com a ajuda da distribuição de materiais promocionais, como a literacia da informação pode ser aplicada a várias disciplinas e, portanto, ser aplicável a muitos cursos. No ano letivo seguinte (2017-2018) metade dos professores solicitaram uma sessão de formação sobre literacia da informação em contexto de sala de aula.

Um dos conteúdos de aprendizagem desenvolvidos pelos bibliotecários do IRSC, e disponibilizado na LibGuide, foi o infográfico RADAR (Neely-Sardon, \& Tignor, 2018) que detalha um plano e uma checklist com perguntas a seguir pelos estudantes para análise e avaliação das fontes de informação (Tabela 2).

Tabela 2. Adaptação do infográfico RADAR criado por Neely-Sardon e Tignor (2018, p. 115)

\begin{tabular}{ll}
\hline Fundamentação & \multicolumn{1}{c}{ Avaliação de fontes de informação com o RADAR } \\
& $\begin{array}{l}\text { Qual o objetivo da informação? Porque foi escrita pelo autor: para informar, apresentar resultados de investigação, vender alguma } \\
\text { coisa? Existe algum tipo de preconceito? São apresentados pontos de vista alternativos? }\end{array}$ \\
\hline Autoridade & $\begin{array}{l}\text { Quais são as credenciais do autor? Quais são as afiliações do autor a organizações, grupos, universidades, etc.? Poder-se-á } \\
\text { encontrar alguma informação sobre o autor? O autor é citado por outras fontes? O editor é bem conceituado? }\end{array}$ \\
\hline Data & $\begin{array}{l}\text { Quando foi a fonte publicada ou atualizada pela última vez? A temática mudou desde essa data? A temática atualiza-se } \\
\text { regularmente, como na tecnologia e na medicina? Onde se situa esta temática no ciclo de informação? }\end{array}$ \\
\hline Precisão & $\begin{array}{l}\text { Existem erros ou declarações que sabemos que são falsas? A fonte é revista pelos pares? A fonte foi revista por um editor ou } \\
\text { especialista? Que outras fontes suportam a informação apresentada? }\end{array}$ \\
\hline Relevância & A informação responde à sua pergunta de investigação? Atende aos requisitos do seu trabalho? Quem é o público-alvo? \\
\hline
\end{tabular}

Foi ainda criado um tutorial interativo que apresenta aos estudantes os critérios de avaliação do RADAR, orientando-os seguidamente na avaliação de uma fake news no navegador, observando cada conjunto de critérios dentro do contexto da página. O LibGuide dos bibliotecários do IRSC também inclui um vídeo que os professores podem usar nas aulas. Pretende-se, no futuro, expandir as sessões de formação para disciplinas ainda não contempladas e consciencializar superiormente, de modo constante, que as competências de literacia da informação são eficazes no combate às fake news e que os bibliotecários têm expertise na matéria, na avaliação de fontes e na verificação de factos (Neely-Sardon, \& Tignor, 2018).

O estudo de Musgrove, Powers, Rebar, e Musgrove (2018) considera que os estudantes universitários são particularmente vulneráveis às fake news, porque é das redes sociais que recebem a maioria da informação online e porque nasceram no tempo da World Wide Web. Por conseguinte, devem ser os bibliotecários e professores a ajudá-los a construir sólidas competências de literacia da informação, reforçando as habilidades de pensamento crítico. Como boas práticas no combate às fake news, os autores sugerem a Framework for Information Literacy for Higher Education, da 
ACRL, a qual apresenta a literacia da informação como um conjunto de habilidades integradas que enlaça a descoberta reflexiva da informação, a compreensão de como a informação é produzida e valorizada, bem como o uso da informação na criação de novo conhecimento e na participação ética nas comunidades de aprendizagem (ACRL, 2016). Musgrove et al (2018) destacam, de um conjunto de seis, uma das frames deste documento de trabalho - a autoridade, que se constrói e é contextual , sendo que a compreensão deste conceito permite que os estudantes examinem criticamente o que são consideradas as evidências, a identificação dos indicadores de autoridade quando associados à informação e a compreensão de que muitas áreas do conhecimento reconhecem autoridades (e.g., oficiais, creditadas, certificadas). Também Pun (2017) sugere o uso desta frame para análise da veracidade da informação. Dado o seu caráter referencial, a ACRL disponibiliza uma sandbox em formato de repositório de acesso aberto que aloja materiais construídos e partilhados por profissionais da informação e que integram as ideias da Framework na formação que providenciam (identificando o conteúdo de cada recurso e associando-o à frame adequada).

Para a identificação das habilidades necessárias para um combate eficaz às fake news por parte das bibliotecas do ensino superior, Musgrove et al (2018) assumem a conjugação de esforços para um resultado mais favorável. Assim, sugerem claramente o uso da ferramenta LibGuide e destacam o trabalho do bibliotecário Eric Novotny (http://guides.libraries.psu.edu/fakenews). O LibGuide é uma aplicação baseada na web e um sistema de gestão de conteúdos usado para criar e organizar guiões eletrónicos. É facilmente incorporada em cursos e sites de bibliotecas e muito acessível aos estudantes online. Os autores destacam, de igual modo, o uso da checklist CRAAP [«tretas»], (Currency, Relevance, Authority, Accuracy, and Purpose), criado por bibliotecários da California State University, que joga linguisticamente com a possibilidade de treinar a deteção de «tretas», e da matriz Who, What, Where, When, Why (Global Digital Citizen Foundation, 2016).

Ainda, a reflexão de Lor (2018) sobre a resposta a dar pelas bibliotecas nos atuais tempos da pós-verdade envolve diversas componentes, a saber:

- As coleções. Investimento na sua construção, na fiabilidade das fontes e recursos de informação, bem como na sua pluralidade de opiniões ideológicas.

- O papel do bibliotecário de referência. Enquanto possuidor de habilidades muito próprias, o bibliotecário de referência encontra a forma criativa de combater as fake news, encoraja o uso de informação fidedigna e estimula o uso de fontes e de bases de dados credíveis.

- A literacia da informação. O advento das fake news levou os profissionais da informação a redobrar os seus esforços na formação de leitores face ao pensamento crítico perante a avaliação das fontes de informação. A infografia da IFLA (2017) é um bom ponto de partida, tendo já sido traduzida em 37 idiomas.

- A recusa das fake news. Na medida em que procurar a verdade pode evidenciar o peso da não-verdade, Lor (2018) destaca o trabalho dos bibliotecários. A verificação da fonte de informação e a correção da desinformação são importantes para a preservação da memória histórica; desta forma, garante-se ao futuro investigador, de forma objetiva, a análise dos acontecimentos atuais sob múltiplos ângulos e abordagens. Trabalhar os dados desta forma requer uma considerável perícia, recursos técnicos e envolve regras e boas práticas - não é um trabalho para amadores. Como exemplo, assinale-se a biblioteca do College of Staten Island (City University of New York) que desenhou um website sobre a melhor forma de ajudar os seus estudantes a detetar fake news, contemplando um tutorial de pesquisa, workshops, um curso sobre a avaliação de fontes de informação, entre outras iniciativas. 
- Recolha de dados. Os bibliotecários são os principais agentes do processo de documentação e preservação dos bancos de dados; deles se espera também um papel interventivo na curadoria destes dados.

Lor (2018) conclui que claramente estas são competências de literacia da informação e que este é o domínio dos profissionais da informação.

Outra reflexão, a de Caridad-Sebastián, Morales-García, Martínez-Cardama e García-López (2018), em formato de revisão da literatura, analisa, entre outras, as iniciativas internacionais propostas pela IFLA e pela ALA, em que se propõe formação com uma componente prática de competências de literacia da informação, a curadoria de dados e de conteúdos, bem como ferramentas de verificação de factos. Neste processo, a biblioteca e os seus profissionais assumem um papel aliado, de advocacia. Propõe-se uma categoria de trabalho, a da referência digital, principalmente para estimular a consciência reflexiva de como, no ensino superior, se pode contrariar o fenómeno da desinformação; representa também uma boa oportunidade para a divulgação de recursos de qualidade subscritos pela biblioteca e oferecer um serviço de referência digital que se estenda para além do tradicional recurso de avaliação das fontes.

A problemática da literacia da informação associada às fake news pode, deste modo, ser assegurada através de sessões de formação, workshops e seminários de opção. Batchelor (2017) destaca a experiência da Universidade de Michigan com um pequeno curso (de um crédito) designado Fake news, lies, and propaganda: how to sort fact from fiction. A avaliação crítica das fontes de informação, através de conteúdos de aprendizagem criados pelos bibliotecários, integra a capacitação formal dos estudantes. Os conteúdos incluem acrónimos e checklists de critérios para a pesquisa e análise da informação antes da seleção de uma fonte, como o CRAAP.

\subsection{Materiais e estratégias da literacia da informação no combate às fake news}

Os estudos selecionados apresentam uma variedade de métodos, iniciativas e projetos que se destacaram positivamente nas suas comunidades e que se elencam (Tabela 3 ).

Tabela 3. Materiais usados pela literacia da informação no combate às fake news

\begin{tabular}{ll}
\hline Bibliotecas & LibGuides \\
(aprendizagem informal) & Tutoriais \\
& Kits de aprendizagem \\
& Workshops \\
& Formação em literacia da informação \\
& Coleços das Bibliotecas (fiabilidade e pertinência) \\
\hline Biblioteca & Framework for Information Literacy for Higher Education (ACRL (2016) \\
(materiais validados) & Infográfico RADAR (Rationale, Authority, Date, Accuracy, Relevance) (Neely-Sardon \& Tignor, \\
& 2018) \\
& Checklist CRAAP (Currency, Relevance, Authority, Accuracy, and Purpose) (Musgrove, Powers, \\
& Rebar, \& Musgrove, 2018) \\
& Matriz Who, What, Where, When, Why (Global Digital Citizen Foundation, 2016) \\
& Additional resources (Cooke, 2018) \\
\hline
\end{tabular}

As estratégias formativas apuradas evidenciam a preocupação dos profissionais da informação face ao crescimento das fake news e à necessidade de intervirem junto das suas comunidades, de forma precisa e pedagógica.

\section{Discussão}


A literacia da informação reserva para si a responsabilidade da formação para o bom uso da informação, garantindo o seu caráter fidedigno no que se refere aos seus conteúdos e à sua produção. A literatura consultada refere que o fator educacional deve necessariamente ser ponderado: a formação de utilizadores motivados para o conhecimento é que lhes permite distinguir a veracidade do que se afirma e identificar qual o cenário para a produção de mais conhecimento (Day, 2017). As pessoas melhor preparadas correspondem a indivíduos que assumem como insuficiente a informação disponibilizada pela Internet. Esta informação deve ser verificada e complementada em diversas fontes, nomeadamente porque o conhecimento também emana das instituições, dos seus métodos e técnicas, o que o torna mais credível e verificável. As bibliotecas do ensino superior são, assim, importantes núcleos neste processo: porque se formam e porque formam (Becker, 2016), mantendo-se atuais e confiáveis e enquanto portais de acesso a estas diversas fontes.

Por conseguinte, um pouco por todo o mundo, e de acordo com a análise dos estudos citados, os profissionais da informação estão preocupados com o progressivo impacto das fake news no capital crítico e científico emanado do ensino superior e estão a realizar intervenções significativas, do ponto de vista pedagógico, preparando-se e estruturando de forma apelativa essa intervenção educacional e formativa. Incidem, entre outros critérios, nas seguintes matérias: o uso de sites de verificação de factos; a leitura de fontes de informação fidedignas; a procura de diferentes perspetivas da informação que levanta dúvidas; o uso de competências de literacia; e a redução da partilha de informação que suscite dúvidas.

Num âmbito mais abrangente, já em 1996 Carl Sagan trabalhou precocemente sobre a ciência e pseudociência, criando o Kit Baloney Detection, em que categorizava os sinais de alerta para as falácias mais comuns, de que se destacam algumas premissas pela sua atualidade:

- Dever-se-ão confirmar os factos, sempre que possível, de forma independente.

- Dever-se-á encorajar o debate das evidências por parte dos defensores de todos os pontos de vista.

- Os argumentos provenientes da autoridade têm pouco peso - em ciência não há autoridades; no máximo, há especialistas.

- Dever-se-á considerar mais do que uma hipótese. Quando algo deve ser explicado, dever-se-á pensar sob todas as diferentes maneiras em que se pode explicar, em que se podem refutar as alternativas. O que sobrevive poderá ser a resposta certa e provavelmente aquela que primeiro suscitou a atenção.

- Não se apegar excessivamente a uma hipótese só porque é nossa; deve ser comparada de forma justa com as alternativas. Quando não se consegue encontrar uma razão para a rejeitar, outros encontrá-la-ão (Sagan, 1996).

Por outro lado, vivendo numa era de tecnologias, as questões relativas à responsabilidade das tecnologias de informação pressupõem o desenvolvimento de melhores algoritmos para melhor permitir a distinção entre o verdadeiro e o falso e para estabilizar os métodos de avaliação da informação, promovendo igualmente a segurança, a estabilidade e a veracidade da informação. Mas, evocando Caridad-Sebastián, Morales-García, Martínez-Cardama e García-López (2018), o combate às fake news não pode ser reservado à tecnologia - é um fator essencialmente humano.

Sugere-se o desenvolvimento de medidas a implementar, quer pelas instituições de ensino superior quer pelos profissionais da informação, para um eficaz combate às fake news, em especial no contexto académico, seio por excelência da formação do pensamento crítico e da investigação aplicada. A análise dos estudos precedentes permite-nos concordar com Buschman (2019, p. 222): 
"libraries help to counter fake news both through specific educative actions aimed at it and by being broadly educative institutions with a coherent notion of their role and relationship to informational discernment in democratic society".

De facto, conhecer outras realidades, ainda que em contextos diferentes, mas tocadas pelos mesmos problemas, inspira-nos a prosseguir a nossa missão primordial de recolher, organizar e tornar acessível a informação, educando os utilizadores para o seu bom uso. Por outras palavras, "librarians and college faculty members are in a unique situation by the validation of their position in the education environment to help students of all ages to develop information literacy and critical thinking skills. A good starting point is to teach students to verify the facts by questioning the source and potential motives" (Musgrove, Powers, Rebar, \& Musgrove, 2018, p. 252). E, dada a frequência com que as fake news circulam, a fiabilidade associada à informação digital é uma matéria pertinente, pelo que os jovens, em breve estudantes universitários e, no futuro, profissionais ou investigadores, devem desde cedo aprender a avaliar criticamente a informação consultada em suporte digital - o problema reside não só na falta de informação e na existência das fake news, mas principalmente nos danos que a desinformação produz em cérebros pouco críticos.

\section{Conclusões}

Os profissionais da informação assumem um compromisso público e pró-ativo de educadores para a promoção de práticas de literacia e de advocacy na sociedade. Na medida em que as competências que adquiriram se vocacionaram para a literacia da informação, estão especialmente habilitados a acompanhar os estudantes do ensino superior no processo de identificação das fake news e na avaliação da informação.

Nos tempos que correm qualquer indivíduo é um consumidor de informação, mas todos os indivíduos devem ter as competências necessárias para, de forma crítica, consumir e criar mais informação, especialmente se se tratarem de estudantes do ensino superior. Sabe-se que as fake news não são controláveis com a censura, mas precisamente com mais informação e com mais formação em fontes de informação. O objetivo é produzir pensadores críticos pró-ativos, investigadores e consumidores de informação que identificam e ultrapassam as fake news e os seus efeitos nefastos.

Concluiu-se que o conhecimento pode resultar em informação, mas a informação não resulta necessariamente em conhecimento - e a informação pode não ultrapassar o patamar da opinião. Os estudos e relatos de experiências já validadas neste âmbito inspiram-nos a prosseguir este desiderato e a contribuir para um melhor e mais ético uso da informação, particularmente no contexto das bibliotecas do ensino superior. Vivemos numa sociedade da informação, mas estamos ainda longe de ser uma sociedade do conhecimento, pelo que importa reforçar estratégias formativas.

Por último, e no espírito da Declaração da IFLA sobre as fake news, o compromisso das bibliotecas de ensino superior e dos seus profissionais é reforçar o seu papel na defesa da importância da liberdade de expressão e da liberdade de acesso à informação.

\section{Referências}

ACRL Research Planning and Review Committee. (2018). 2018 Top trends in academic libraries: A review of the trends and issues affecting academic libraries in higher education. College \& Research Library News, 79(6), 286-293,300. doi: 10.5860/crln.79.6.286 
American Library Association [ALA]. (2017). Resolution on access to accurate information. Chicago: Author. Retrieved from

http://www.ala.org/advocacy/intfreedom/statementspols/ifresolutions/accurateinformation

Association of College and Research Libraries [ACRL]. (2016). Framework for information literacy for higher education. Chicago, IL: American Library Association. Retrieved from http://www.ala.org/acrl/standards/ilframework

Batchelor, O. (2017). Getting out the truth: The role of libraries in the fight against fake news. Reference Services Review, 45(2), 143-148. doi: 10.1108/RSR-03-2017-0006

Becker, B. W. (2016). The librarian's information war. Behavioral \& Social Sciences Librarian, 35(4), 188-191. doi: 10.1080/01639269.2016.1284525

Caridad-Sebastián, M., Morales-García, A. M., Martínez-Cardama, S., \& García-López, F. (2018). Infomediación y posverdad: El papel de las bibliotecas. El Profesional de la Información, 27(4), 891-898. doi: 10.3145/epi.2018.jul.17

Cid-Leal, P., \& Perpinyà-Morera, R. (2015). Competència informacional en traducció: Anàlisi dels hàbits dels estudiants universitaris en la consulta i l'ús de fonts d'informació. BiD: textos universitaris de biblioteconomia i documentació, (34). doi: 10.1344/BiD2015.34.10

Comber, B., \& Grant, H. (2018). Working critically and creatively with fake news. Journal of Adolescent and Adult Literacy, 62(3), 329-332. doi: 10.1002/jaal.905

Comissão Europeia. (2018). Combater a desinformação em linha: uma estratégia europeia [COM (2018) 236 final]. Bruxelas: Autor.

Cooke, N. (2018). Fake news and alternative facts: Information literacy in a post-truth era. Chicago, IL: American Library Association.

Connaway, L. S., Julien, H., Seadle, M., \& Kasprak, A. (2017). Digital literacy in the era of fake news: Key roles for information professionals. ASIS\&T, 54(1), 554-555. doi: 10.1002/pra.2.2017.14505401070

Day, R. E. (2017). Before information literacy [or, who am I, as a subject-of-(information)-need?]. ASIS\&T, 54(1), 57-60. doi: 10.1002/pra2.2017.14505401007

Finley, W., McGowan, B., \& Kluever, J. (2017). Fake news: An opportunity for real librarianship. ILA Reporter, 35(3), 8-11.

Fontanin, M. (2018). Con il pretesto delle false notizie: Insegnare il pensiero critico nella scuola italiana a partire da Carol C. Kuhlthau. AIB studi, 58(2), 267-283. doi: 10.2426/aibstudi-11825

Forbes, S., \& Mintz, A. P. (2002). Web of deception: Misinformation on the Internet. Medford: Information Today. 
Gilchrist, A. (2018). Post-truth: An outline review of the issues and what is being done to combat it. IBERSID, 12(2), 13-24.

Global Digital Citizen Foundation. (2016). The ultimate cheatsheet for critical thinking. Retrieved from https://i1.wp.com/globaldigitalcitizen.org/wp-content/uploads/2016/12/ultimate-criticalthinking-worksheet.jpg?ss1\%20\&hx003D;1

Horowitz, J. (2017 October 18). In Italian schools, reading, writing and recognizing fake news. New York Times. Retrieved from https://www.nytimes.com/2017/10/18/world/europe/italy-fakenews.html

International Federation of Libraries Association [IFLA]. (2018). IFLA trend report: 2018 update. The Hague: Author. Retrieved from http://trends.ifla.org/update-2018

Jasanoff, S. (2016). The ethics of invention: Technology and the human future. New York: WW Norton.

Johnson, B. (2017). Information literacy is dead: The role of libraries in a post-truth world. Computers in Libraries, 37(2).

LIBER Europe. (2017). Research libraries powering sustainable knowledge in the digital age: Strategy 2018-2022. The Hague: Author. Retrieved from https://libereurope.eu/wpcontent/uploads/2017/05/May2017Mail_LIBER-Strategy-2018-2022.pdf

López-Borrull, A., Vives-Gràcia, J., \& Badell, J. I. (2018). Fake news: Amenaza u oportunidad para los profesionales de la información y la documentación? El Profesional de la Información, 27(6), 1346-1356. doi: 10.3145/epi.2018.nov.17

Lor, P. J. (2018). Democracy, information, and libraries in a time of post-truth discourse. Library Management, 39(5), 307-321. doi: 10.1108/LM-06-2017-0061

Mintz, A. P. (2012). Web of deceit: Misinformation and manipulation in the age of social media. Medford: Information Today.

Musgrove, A. T., Powers, J. R., Rebar, L. C., \& Musgrove, G. J. (2018). Real or fake? Resources for teaching college students how to identify fake news. College \& Undergraduate Libraries, 25(3), 243-260. doi: 10.1080/10691316.2018.1480444

National Literacy Trust. (2018). Fake news and critical literacy: The final report of the Commission on Fake News and the Teaching of Critical Literacy in Schools. London: Author.

Neely-Sardon, A., \& Tignor, M. (2018). Focus on the facts: A news and information literacy instructional program. Reference Librarian, 59(3), 108-121. doi:

10.1080/02763877.2018.1468849

Pun, R. (2017). Hacking the research library: Wikipedia, Trump, and information literacy in the Escape Room at Fresno State. Library Quarterly, 87(4), 330-336. doi: 10.1086/693489 
Rose-Wiles, L. (2018). Reflections on fake news, librarians, and undergraduate research. Reference \& User Services Quarterly, 57(3), 200-204.

Sagan, C. (1996). The fine art of baloney detection. In: The Demon-Haunted World [seminar]. Retrieved from http://www.inf.fu-berlin.de/lehre/pmo/eng/Sagan-Baloney.pdf

UNESCO. (2018). Journalism 'fake news' and disinformation. Paris: UNESCO.

Vosoughi, S., Roy, D., \& Aral, S. (2018). The spread of true and false news online. Science, 359(6380), 1146-1151. doi: 10.1126/science.aap9559 